\title{
TOPOLOGICAL STRUCTURES OF COMPLEX BELIEF SYSTEMS
}

\author{
J. Nescolarde-Selva \\ Department of Applied Mathematics. University of Alicante. Alicante. Spain. \\ J.L. Usó-Doménech \\ Department of Mathematics. University Jaume I. Castelló de la Plana. Spain.
}

\begin{abstract}
The concepts of substantive beliefs and derived beliefs are defined, a set of substantive beliefs S like open set and the neighbourhood of an element substantive belief. A semantic operation of conjunction is defined with a structure of an Abelian group. Mathematical structures exist such as poset beliefs and joinsemilatttice beliefs. A metric space of beliefs and the distance of belief depending on the believer are defined. The concepts of closed and opened ball are defined. $S^{\prime}$ is defined as subgroup of the metric space of beliefs $\Sigma$ and $\mathrm{S}^{\prime}$ is a totally limited set. The term $\mathrm{s}$ is defined (substantive belief) in terms of closing of S.' It is deduced that $\Sigma$ is paracompact due to Stone's Theorem. The pseudometric space of beliefs is defined to show how the metric of the nonbelieving subject has a topological space like a nonmaterial abstract ideal space formed in the mind of the believing subject, fulfilling the conditions of Kuratowski axioms of closure. In order to establish patterns of materialization of beliefs we are going to consider that these have defined mathematical structures. This will allow us to understand better cultural processes of text, architecture, norms, and education that are forms or the materialization of an ideology. This materialization is the conversion by means of certain mathematical correspondences, of an abstract set whose elements are beliefs or ideas, in an impure set whose elements are material or energetic. Text is a materialization of ideology.
\end{abstract}

Keywords: Belief materialization, Belief system, Connotative significance, Derived beliefs, Structurating structure, Substantive beliefs.

\section{INTRODUCTION}

We know that the human being is a social animal. This is a common fact. Moreover, the human being is defined as a rational being. It is clear and nobody can deny that human creations include logic, mathematics, philosophy, science, and jurisprudence. These are all products of rationality or abstract thought. Nevertheless, human sociability goes further that the sociability of an animal herd. Societies were founded, cohere, develop, degenerate and die based on their belief systems.

Reason cannot prove the beliefs it is based upon. Beliefs arise through experience. Experience need previous beliefs and reason to be assimilated, and reason needs experience to be formed, as beliefs need reason as well. Beliefs, reason and experience, are based upon each other. Context is dynamic, and formed upon beliefs, reason and experience. This where relative understanding lies. Since relative understanding is independent of our context, it is also dependant on our beliefs, reasoning, and experiences. Contexts are dynamic because they are changing constantly as we have new experiences and change our beliefs and our ways of reasoning. Belief systems (Borhek and Curtis, 1983) are structures of norms that are interrelated and that vary mainly in the degree in which they are systemic. What is systemic in the Belief system is the interrelation between several beliefs.

Perceived Reality is constructed by means of systems of signs, being affected and being changed by means of Belief systems. Peirce (1958) demonstrates that the semiotic process has been half-full culturally, that is to say, within a certain Belief system. A subject cannot understand a sign without talking about to a system that is learned socially and that allows him to make sense of perception. In the same way, the classification of signs in closed typologies can be deceptive, since the status of the sign depends strongly on the form in which the sign is used within the Belief system. A 
significant can nevertheless be iconic in a belief context and, to be symbolic in another context.

Moreover, these signs are not rational. The species Homo sapiens developed so-called belief systems. These are sets of beliefs reinforced by culture, theology, experience and training as to how the world works, cultural values, stereotypes, political viewpoints, etc. In agreement with the Spanish philosopher Ortega y Gasset "in beliefs we live, we move and we are [... ] the beliefs constitute the base of our life, the land on which we live [... ] All our conduct, including the intellectual life, depends on the system of our authentic beliefs. In them [... ] lies latent, as implications of whatever specifically we do or we think [... ] the man, at heart, is believing or, which is equal, the deepest stratum of our life, the spirit that maintains and carries all the others, is formed by beliefs..”. Beliefs are often considered as convictions or as religious beliefs, but as scientists, there are also philosophical beliefs relating to the sphere of daily life. If a stimulus is received, it may be interpreted through the belief system to be whatever the belief system might lead the recipient to rationalize. A belief system need have no basis in reality so long as it consistently provides adequate explanations. It takes us to define a human being like Homo religious.

Claude Levi-Strauss (1963) argued that structural factors determine our cultural expressions so as to make them resonate with us beneath awareness. His explanatory strategy first involved reducing expressive objects (e.g., artwork or mythological stories) to contrastive structures in which some elements were opposed to others. These structures were then argued to be similar in form to (or otherwise influenced by) an abstract picture of the social structure in which they were produced. The formal correspondence produced a resonance that explained why particular expressive objects were enjoyed and repetitively interpreted elements. Substantively, Levi-Strauss followed Durkheim's suggestion in The Elementary Forms of the Religious Life (Durkheim, 2001) and in Primitive Classification (Durkheim and Mauss, 1963) that certain cognitive constructs have the same form as elements of social life. Levi-Strauss argued that "savage minds" employed different principles in constructing myths from those we use in stories - ones that were entirely novel and heretofore unimagined. In his decoding of myths, the reduction to contrastive structures was retained, but the explanation of their pattern took a path similar to the generative grammar being formulated in linguistics by Noam Chomsky, looking to features of the human brain rather than social structure.

There is no consensus in the literature about the logical form of a belief system (Andreas, 2011). In fact, few attempts have been made to characterize belief bases by formal, logical means. Rott (2001) and Hansson (1999) introduce the notion of a belief system such that it contains only non-derived beliefs. A sentence - is thus an element of the belief base $\mathrm{H}$ if and only if - it is non-derived and accepted. The investigation of base revisions with the intent of an axiomatic characterization with postulates has been proposed by Hansson. A belief base $\mathrm{H}$ may be joined with a set $\mathrm{E}$ of axioms belonging to some background theory. This strategy has been studied by Rott (2001) and Brewka (1991) in a systematic way. There, the axioms of background theories need only be defeasibly valid ${ }^{1}$. They are simply called expectations, which give rise to the use of $\mathrm{E}$ as the symbol for the set of axioms of potentially relevant background theories. Rott's

\footnotetext{
${ }^{1}$ Defeasibly valid means an argument is rationally compelling but not deductively valid.
} 
investigation of base revisions in the context of expectations will prove highly useful for the present attempt at an integration of the structuralist framework into belief revision theory

\subsection{Mythical and religious beliefs}

Man has lived in two environments: one natural and the other supernatural (Swanson, 1964). The structures of both worlds vary greatly in human experience. In the words of the author- towards man, the supernatural may be indifferent, spiteful, wantonly malevolent, supportive, supervisory, distant, intimate, transcendent, or immanent. Toward the supernatural - man may be scornful, friendly, fearful, awe-struck, manipulative, indifferent, submissive, reverent, joyful, aggressive, or loving.

No procedure of empirical science allows us to determine with absolute certainty that an event $\mathrm{A}$ is the cause of other event $\mathrm{B}$. What one can sometimes show is that B always appears after A and that B appears only when $A$ is present. We can never be certain that $A$ and $B$ will have this relationship under all possible conditions because we are able to study them in only a limited number of situations. We can never be certain that it is A, as such, rather than some aspect of A or something which always accompanies A without being a part of it, which is the necessary and sufficient antecedent of $\mathrm{B}$. The confidence that $\mathrm{A}$ is the case of $\mathrm{B}$ is increased by several factors:

1) There is causal relation if we have some logically valid reasons for thinking that it should.

2) If the relationship appears under a wide variety of conditions.

3) If alternative explanations may be discarded as contrary to empirical observations.

4) If we are able to control the appearance of antecedent conditions other than A so that $\mathrm{A}$ alone seems to precede $\mathrm{B}$.

Although absolute empirical proof of any positive assertion about causality is out of the question, absolute empirical disproof is often quite possible: B appears in the absence of A or A is not always followed by B. It is a curious fact of human nature that we can be absolutely certain that something is not true, but only more or less certain that something is true (Swanson, 1964). Two theories have been formulated for the explanation of the origin of beliefs:

1) The experiences and inferences of prehistoric men. Knowledge about beliefs shows that they do not persist by themselves. An idea, attitude or belief must correspond to current experiences with the environment if it is to continue across the generations. As the result, we may expect that forces which produce and support current beliefs are present along with those beliefs.

2) Direct experiences with $\mathrm{Mana}^{2}$ and spirits. By definition, these supernatural entities stand apart from the natural universe, freed of its laws and limitations and we are not able to observe them through the instruments of nature. Behind nature events lies the supernatural, that it to say, a realm of potentialities and purposes of which natural events are concretions or expressions in the same way

\footnotetext{
${ }^{2}$ Mana are elemental spirits
} 
as human behaviors are expressions of potentialities and purposes held by the men who produces them. Mana represents the potentialities which underlie nature and spirits represent organized clusters of the underlying purposes (Swanson, 1964). When he is confined to the world of nature, man is unable to produce what he wants merely by having the desire to do so, by informing the natural order with his purposes. He must create changes in the material universe which, of themselves, produce yet other changes until his objective is reached. At no point do his ideas or purposes intervene to change the environment. They must be implemented by material action in the material world or that world remains as it was. Supernatural forces are free of these limitations imposed on natural action in the material world. Not only does supernatural force have powers not given to men, but, unless opposed by other and stronger spirits or by magic, the ends toward which those forces are directed are always accomplished. The supernatural powers are immortal. They neither die nor become impotent with age. Possibly the mystery of death leads to the first belief: the existence of a immortal spirit, in the man and other living beings. The nature of life, of sleep, of death, and of dreams was the stuff which inspired religious thought. Reflecting on these mysteries, man developed the distinction between the human body and the spirit dwelling within it.

By symbols Geertz (1973) meant a carrier embodying a conception as he saw religion and culture as systems of communication. Eliade (1978) suggested that the earliest document in the history of beliefs is located in the symbolism of stone tools or tool making. For Harrod (1992) the first technological discoveries not only insured the survival and development of the human species: they also produced a universe of mythical-religious values and inspired and fed the creative imagination. Human religious thought and moral values clearly rest on a cognitive-linguistic base. Fundamental to humanity, as inseparable as language, is the common origin of religion and art. Even in primitive less figurative art works and more clearly in art with religious content, the primitive artist is the creator of a message; he exerts through the forms a symbolized function that is also evident in music, dance and language. This message indicates the physical and psychic necessity to provide to the individual and the social group the importance of understanding the universe. To provide a place for man, by means of the symbolic apparatus, in the movable and random world that surrounds him. One suggestion has the gods representing the sun and the goddesses the moon. Another would have us see gods personifying a life-force causing plants, especially the foodplants, to grow anew each spring (Walsby, 1947). These proposed explanations, and others attempting to trace the origins of the divinities in nature, do much to account for the pattern of the rise, the decline and the renascence so common in religious myths, and for the emotions associated with religion. But although sun, moon and stars undeniably play a part, they serve less as an origin of the religious impulse as a way of providing it with a local habitation. Cows and cats also have provided shapes for divinity to occupy, but few propose these creatures have a role as a source of religious belief. There is more to religion than these explanations can explain, and - what concerns us here - they fall short of accounting for the omnipotence credited to the supreme deities.

Geertz (1973) saw religion as one of the cultural systems of a society. He defined religion as a system of symbols which acts to establish powerful, pervasive and longlasting moods and motivations in men by formulating conceptions of a general order of existence and clothing these conceptions with such an aura of factuality that the moods 
and motivations seem uniquely realistic. The concept Religion is sometimes used interchangeably with that of belief system, but it is more socially defined than personal convictions, and it entails specific behaviors. Nevertheless belief systems may not necessarily refer to a religion, though a religion may be referred to as a belief system. Religion is a system of human thought which usually includes a set of narratives, symbols, beliefs and practices that give meaning to the practitioner's experiences of life through reference to a higher power or ultimate truth.

Religion may focus on specific supernatural, metaphysical, and moral claims about Reality which may yield a set of deontical norms, values, and a particular lifestyle. Religion as we know it today is an integral part of civilization, influential, immensely complex and deeply enmeshed in social life. Political movements turn out on examination to form a significant series and one might have expected religions to fall into a corresponding distribution, but they do not. Religion symbolizes the strength and cohesiveness of society, but no society, simple or sophisticated, provides any model for unlimited power; every society acts within limitations imposed either by the natural world or by other societies. Religious behavior, the religiosity, is not made up only of religion, but that it supposes, everything in a set of physiological and psychological facts that generate an emotional field in which the rational explanation does not occupy the primary position.

\subsection{Initial hypotheses}

Considered these arguments we propose following initial hypotheses:

Hypothesis 1: Beliefs are not products of reason or of abstract and logical thought.

Hypothesis 2: In the origin of any belief system there is always a supernatural system of beliefs.

Hypothesis 3: Derived beliefs become substantial beliefs with the passage of time, giving origin to a more or less ample body of substantive beliefs, that is to say, a religion.

Hypothesis 4: When belonging to the Ideological Doxical Superstructure (NescolardeSelva and Usó-Domènech. 2013 $3^{a, b, c}$, Usó-Domènech and Nescolarde-Selva, 2012), the set $S$ of substantive beliefs will be "ideal", that is to say, merely abstract.

Hypothesis 5: Substantive and derived sets form a graphed text having a topological structure which represents the way in which the individual organizes semantic content, concepts and propositions in his cognitive structure through subsumption, differentiation and integration.

\section{SUBSTANTIVE AND DERIVED BELIEFS}

A belief system (BS) is a set of related ideas, learned and shared which has some permanence in time and space, and to which individuals and/or groups exhibits some commitment (Borhek and Curtis, 1983; Usó-Doménech and Nescolarde-Selva, 2012). The conditions of permanence, commitment, and connectedness are variable characteristics through which we expect belief systems to be related to social organization. Any belief system will be formed by two essential levels:

1) Ideal or abstract level. 
2) Material level or text.

The first of the levels or the abstract level of the BS it is the reason for this work. It is embedded in the individual mind and has been acquired by means of a physical transmission, either oral or visual, through a textual materialization, such as a written, pictorial, architectural, musical, etc, text. We may in fact affirm that a belief system operates as a cybernetic feedback process (Figure 1).

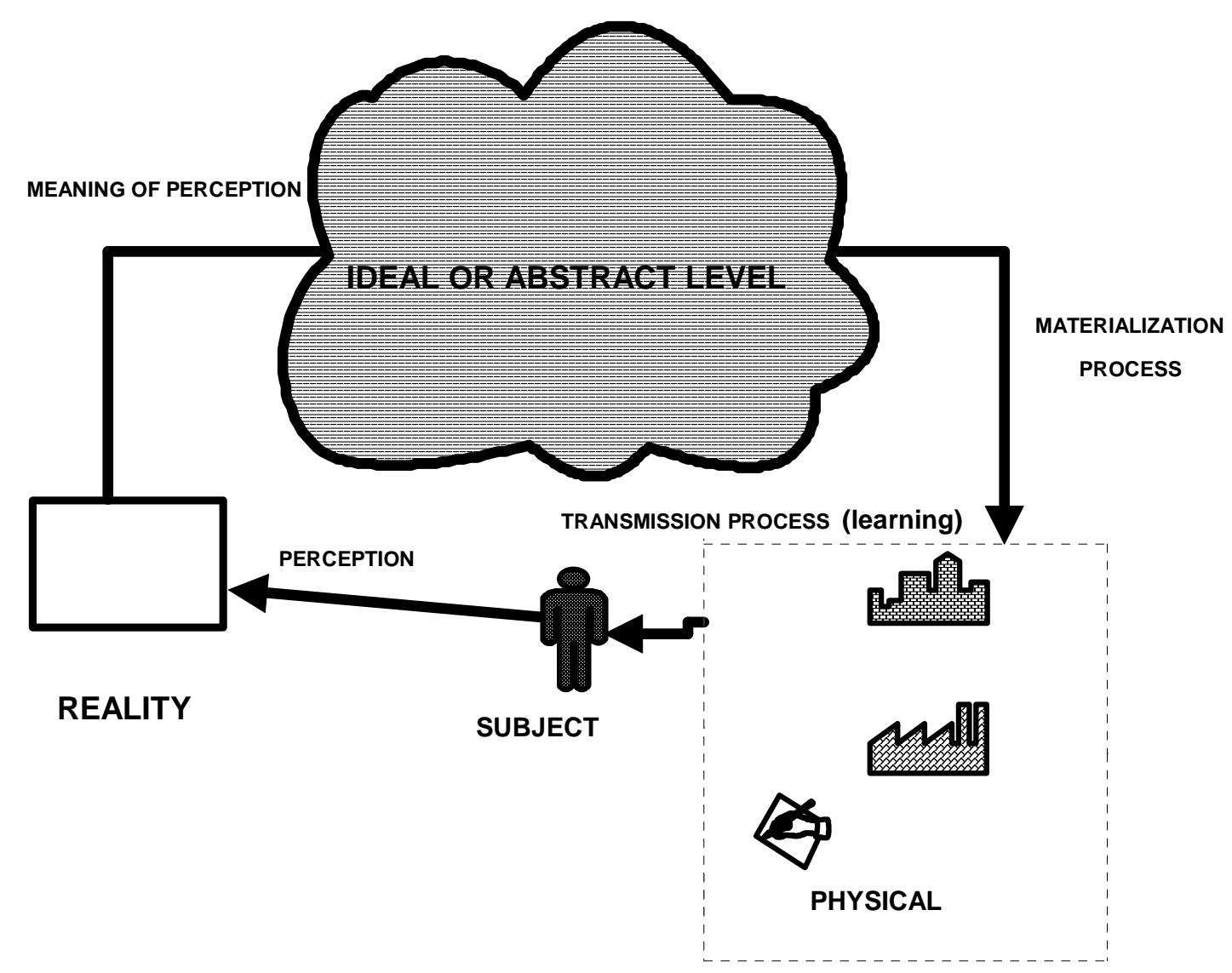

Figure 1: Belief system as a cybernetic feedback process.

Definition 1: The abstract belief level $(B S)$ is formed by a set of elements denominated substantive beliefs $\mathrm{S}$ forming the unquestionable truths of the system (axioms) and a set of derived beliefs $D$, formed from substantive beliefs.

Substantive beliefs constitute the axioms of the system, while many of derived beliefs will constitute their theorems.

Example 1: In the same sense as Christianity or Islam, Judaism, cannot be credited with the possession of Articles of Faith. Many attempts have indeed been made at systematizing and reducing to a fixed phraseology and sequence the contents of the Jewish religion (Scholem, 1941). However, these have always lacked one essential element: authoritative sanction on the part of a supreme ecclesiastical body. In addition, for this reason they have not been recognized as final or regarded as having universally binding force. However, to a certain extent incorporated in the liturgy and utilized for purposes of instruction, these formulations of the cardinal tenets of Judaism carried no greater weight than that imparted to them by the fame and scholarship of their 
respective authors. None of them had a character analogous to that given in the Church to its three great formulas (the so-called Apostles' Creed, the Nicene or Constantopolitan Creed, and the Athanasian Creed), or even to the Kalimat AsShahadat of the Muslims. None of the many summaries from the pens of Jewish philosophers and rabbis have been invested with similar importance and prominence. The reasons for this relative absence of official and obligatory creeds are easily ascertained. The most widely spread and popular of all creeds is that of Maimonides, embracing the thirteen articles. Why he chose this particular number has been a subject of much discussion. Some have seen in the number a reference to the thirteen attributes of God. Probably the choice of the number has no significance. His articles are:

$\mathbf{s}_{\mathbf{1}}$ Principle I: To know the existence of the Creator.

$\mathbf{s}_{2}$ Principle II: The unity of God.

$\mathbf{s}_{3}$ Principle III: The denial of physicality in connection with God.

$\mathbf{s}_{\mathbf{4}}$ Principle IV: God's Antiquity.

$\mathbf{s}_{5}$ Principle V: That God, blessed be He, is worthy that we serve Him, to

Glorify Him, to make known His greatness, and to do His

Commands.

$\mathbf{s}_{\mathbf{6}}$ Principle VI: Prophecy.

$\mathbf{s}_{7}$ Principle VII: The prophetic capacity of Moses our Teacher, peace be upon him.

s8 Principle VIII: That the Torah is from heaven [God].

S9 Principle IX: The completeness of the Torah.

$\mathbf{S}_{10}$ Principle X: That God knows man's actions and does not remove His eye from them.

$\mathbf{S}_{11}$ Principle XI: That God gives reward to he who does the commandments of the Torah and punishes those that transgress its admonishments and warnings.

$\mathbf{S}_{12}$ Principle XII: The era of the Messiah.

$\mathbf{s}_{13}$ Principle XIII: Resurrection of the dead.

In the section are exposed the main elementary mathematical structures (Usó-Doménech and Nescolarde-Selva, 2012).

Let $S=\left\{s_{1}, s_{2}, \ldots, s_{n}\right\}$ be the set of substantive beliefs and $D=\left\{d_{1}, d_{2}, \ldots, d_{m}\right\}$ the set of derived beliefs, such that $B S=S \cup D=\left\{s_{1}, s_{2}, \ldots, s_{n}, d_{1}, d_{2}, \ldots, d_{m}\right\}$. There is the no belief or empty belief that we will represent by $\varnothing$. Set BS forms a belief sequence because it is an ordered list of objects. It contains terms or beliefs, and the number of terms is called the length of the sequence. Order matters, and the exact same terms can appear multiple times at different positions in the belief sequence. BS forms a finite sequence with terms in the set BS because it is a function from $\left\{s_{1}, s_{2}, \ldots, s_{n}, d_{1}, d_{2}, \ldots, d_{m}\right\}$ to BS.

Due to the complexity of the belief systems, we have thought that it is advisable to limit this approach to the study of the set of substantive beliefs $S$, avoiding the derived beliefs D (Usó-Doménech and Nescolarde-Selva, 2012). Mathematical structures of beliefs are based on Klüver (2011), Anderson (1987), Birkhoff (1967), Bourbaki (1972), Bryant (1985), Burris and Sankappanavar (1981), Kelley (1955) and Willard (1970).

\subsection{Set characteristics}


Let $S=\left\{s_{1}, s_{2}, \ldots, s_{i}, \ldots, s_{n}\right\}$ be a set of substantives beliefs ${ }^{3}$. Set $\mathrm{S}$ has the following characteristics:

1) The set $\mathrm{S}$ is a countable set because there exists an injective function $f: S \rightarrow N$ being $\mathrm{N}$ the natural numbers.

2) The set $S$ is bounded because it has both upper and lower bounds.

3) In all sets of substantive beliefs $S$ there exists one substantive term which we will consider as the main term.

Example 2: The Maimonides' Creed has a longitude of 13.

The main term is $\mathbf{s}_{\mathbf{1}}=$ To know the existence of the Creator.

Term $\mathrm{s}_{5}$ Principle V: That God, blessed be He, is worthy that we serve Him, to glorify Him, to make known His greatness, and to do His commands. Can be placed in subsentences or subterms:

$\mathrm{s}_{51}=\varepsilon_{1}=$ That God

$\mathrm{S}_{52}=\varepsilon_{2}=$ blessed be He

$\mathrm{s}_{53}=\varepsilon_{3}=i$ is worthy that we serve Him

$\mathrm{s}_{54}=\varepsilon_{4}=$ to glorify Him

$\mathrm{s}_{55}=\varepsilon_{5}=$ to make known His greatness

$\mathrm{s}_{56}=\varepsilon_{6}=$ and to do His commands.

Let $\mathbf{L}$ be a language. We suppose the existence of $\mathrm{n}$ substantive beliefs $s_{1}, s_{2}, \ldots, s_{n}$ coexisting at a certain historical moment. Let $\beth$ be the set of all substantive beliefs such that $\boldsymbol{\beth}=\left\{s_{1}, s_{2}, \ldots \ldots ., s_{n}, \ldots ..\right\}$. Let $\varepsilon$ be a sentence such that $\varepsilon \in s_{i}, s_{i} \in \boldsymbol{\beth}$. Let be (+) the operation of adding a sentence and (- ) the operation of clearing a sentence. Then

Definition 2: A set of substantive beliefs $S \subset \mathbf{Z}$ is called open, if for each $s \in S$ there exists and $\varepsilon \neq \varnothing$ such that the interval $(s-\varepsilon, s+\varepsilon)$ is contained in $S$.

Definition 3: A set $S$ of substantive beliefs is called closed if the complement of $S, B S \backslash$ $S$, is open.

Closed sets S correspond to belief systems ideologically closed and impermeable, such as dogmatic religions or political totalitarian ideologies.

Definition 4: In an open $S$ interval, $(s-\varepsilon, s+\varepsilon)$ is called a neighborhood of term $s$.

Let $S=s_{1}, s_{2}, \ldots, s_{n}$ be a collection of substantive beliefs (axioms). We define the operation $\underset{\text { sem }}{\wedge}$ or semantic conjunction. We define the following properties:

\footnotetext{
${ }^{3}$ Over time, some derived beliefs become substantive beliefs. At the same time, some substantive beliefs change their order in the set of substantive beliefs or simply disappear. This indicates the existence of a dynamic within this set.
} 
1) For the believer, each substantive belief will have a truth value equal to 1 , $v(s)=1$.

2) There is the absolute negation of a substantive belief $\neg s_{i}$ with truth value equal to $0 v\left(\neg s_{i}\right)=0$.

3) The semantic conjunction between two or more substantive beliefs will have a truth value equal to $1 v\left(s_{1} \underset{\text { sem }}{\wedge} s_{2}\right)=1$.

4) An empty substantive belief exists $\varnothing$.

The pair $(S, \underset{\text { sem }}{\wedge})$ has the following properties:
1) Closure: $\forall s_{1}, s_{2} \in S,\left(S_{1} \underset{\text { sem }}{\wedge} S_{2}\right) \in S$
2) Associativity: $\forall s_{1}, s_{2}, s_{3} \in S,\left(s_{1} \underset{\text { sem }}{\wedge} s_{2}\right) \underset{\text { sem }}{\wedge} s_{3}=s_{1} \underset{\text { sem }}{\wedge}\left(s_{2} \underset{\text { sem }}{\wedge} s_{3}\right)$

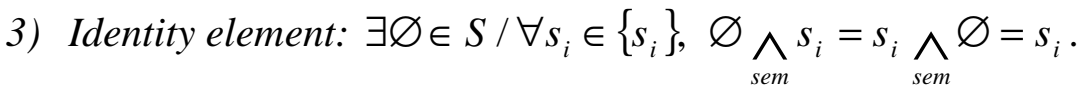
4) Inverse element: $\forall s_{i} \in S, \exists \neg s_{i} \in S / s_{i} \bigwedge_{\text {sem }} \neg s_{i}=\neg s_{i} \bigwedge_{\text {sem }} s_{i}=\varnothing$
5) Commutativity: $\forall s_{1}, s_{2} \in S / s_{1} \underset{\text { sem }}{\wedge} s_{2}=s_{2} \underset{\text { sem }}{\wedge} s_{1}$

Therefore $(S, \underset{\text { sem }}{\wedge})$ is an abelian group. For the case of a believer, the identity element and inverse element suppose processes of conversion or abandonment of the belief respectively.

\subsection{The poset belief}

In a set of substantive beliefs $\mathrm{S}$ exist a partial order relation $\geq$ or "priority relation" which is:

1) Reflexive: $s_{1} \geq s_{1}$.

2) Antisymmetric: If $s_{1} \geq s_{2}$ and $s_{2} \geq s_{1}$ then $s_{1}=s_{2}$.

3) Transitive: If $s_{1} \geq s_{2}$ and $s_{2} \geq s_{3}$ then $s_{1} \geq s_{3}$.

4) Totality: $\forall s_{1}, s_{2} \in S, s_{1} \geq s_{2} \vee s_{2} \geq s_{1}$

In other words, a priority order over $\mathrm{S}$ is an antisymmetric preorder, having the following characteristics:

1) The number of terms of $S$ is finite. Therefore when $(S, \geq)$ a finite partial order relation or belief poset.

2) Let $s_{1}, s_{2}, s_{3}$ be three terms of $(\mathrm{S}, \geq)$ such that $s_{1} \geq s_{2} \geq s_{3}$. The element $s_{1}$ is the belief term join, supremum belief or least upper belief bound of $\mathrm{S}$ if the following conditions are satisfied:

a) $s_{1} \geq s_{2}$ and $s_{2} \geq s_{3}$.

b) $\forall s_{i}, s_{j}, s_{l} \in S$ such that $s_{1} \geq s_{i}$ and $s_{1} \geq s_{j}$ we have $s_{1} \geq s_{l}$. 
Consequence 1: The set $S$ is a directed set because it has together with a reflexive and transitive binary relation $\geq$, with the additional property that every pair of elements has an upper bound.

Note 1: The supremum belief is the main term of the set of substantive beliefs.

Note 2: The supremum belief is the greatest element of the belief poset.

Condition 1: $(S, \geq)$ has always a belief term join or supremum belief.

\subsection{The belief term join-semilattice}

As the belief term join does always exist, it is denoted $s_{i} \vee s_{j}$. If all pairs of terms of $\mathrm{S}$ have belief term joins, then indeed the belief term join is a binary operation on $S$, and it is easy to see that this operation fulfils the following three conditions: For any terms $s_{1}, s_{2}, s_{3}$ in $S$ :
A1) Commutativity: $s_{1} \vee s_{2}=s_{2} \vee s_{1}$.
A2) Associativity: $s_{1} \vee\left(s_{2} \vee s_{2}\right)=\left(s_{1} \vee s_{2}\right) \vee s_{3}$.
A3) Idempotence: $s_{1} \vee s_{1}=s_{1}$.

In a set of substantive beliefs $S$ with a partial order priority relation (belief poset), the belief term join is unique. We suppose that $s_{1}, s_{1}^{*}$ are both belief term joins of $\mathrm{S}$. Then $s_{1} \geq s_{1}^{*} \geq s_{1}$ whence indeed $s_{1}=s_{1}^{*}$. And a set $\mathrm{S}$ of substantive beliefs is a closed set, not allowing the existence of more terms. If another term as $s_{1}{ }^{*}$ existed it would be a different substantive belief set, which is not allowed by the same definition of substantive belief set.

Definition 5: The binary priority operation $\geq$ on a substantive belief set $S$ is a belief term join, if it satisfies the three conditions $A 1, A 2$, and A3 supra and the pair $(S, \geq)$ then is a belief join-semilattice.

\subsection{The belief term meet-semilattice}

Let $S$ be a substantive belief set with a partial priority order $\geq$, and let $s_{i}$ and $s_{j}$ be two terms in $S$. A term $s_{n}$ of $S$ is the belief term meet or infimum belief of $s_{i}$ and $s_{j}$, if the following two conditions are satisfied:

1) $s_{i} \geq s_{n}$ and $s_{j} \geq s_{n}$.

2) for any $s_{n+1}$ in $S$, such that $s_{i} \geq s_{n+1}$ and we have $s_{j} \geq s_{n+1}$.

A belief term meet of $s_{i}$ and $s_{j}$ is unique, since if both $s_{n}$ and $s_{n}{ }^{\prime}$ are greatest lower bounds of $s_{i}$ and $s_{j}$, then $s_{n} \geq s_{n}{ }^{\prime} \geq s_{n}$, whence indeed $s_{n}=s_{n}{ }^{\prime}$.

Note 3: A belief term meet will exist not always in a belief poset. 
If the belief term meet does exist, it is denoted $s_{i} \wedge s_{j}$. If all pairs of terms have belief term meets, then indeed the belief term meet is a binary operation on $S$. For any elements $s_{1}, s_{2}, s_{3} \in S$ this operation fulfils the following three conditions:

B1) Commutativity: $s_{1} \wedge s_{2}=s_{2} \wedge s_{1}$.

B2) $s_{2} \geq s_{1}$ Associativity: $s_{1} \wedge\left(s_{2} \wedge s_{2}\right)=\left(s_{1} \wedge s_{2}\right) \wedge s_{3}$.

B3) Idempotence: $s_{1} \wedge s_{1}=s_{1}$.

We then may define a binary priority relation on $\mathrm{S}$, by stating that $s_{1} \geq s_{2}$ if $s_{2} \vee s_{1}=s_{1}$. In fact, this relation is a partial order on $\mathrm{S}$. Indeed, for any elements $s_{1}, s_{2}, s_{3}$ in $\mathrm{S}$ :

C1) $s_{1} \geq s_{1}$, since $s_{1} \vee s_{1}=s_{1}$ by A3.

C2) If $s_{1} \geq s_{2}$ and $s_{2} \geq s_{1}$, then $s_{1}=s_{2} \vee s_{1}=s_{1} \vee s_{2}=s_{2}$ by A1.

C3)If $\quad s_{2} \geq s_{3} \quad$ and $\quad s_{1} \geq s_{2}$ then $s_{1} \geq s_{3}$, since then $s_{3} \vee s_{1}=s_{3} \vee\left(s_{2} \vee s_{1}\right)=\left(s_{3} \vee s_{2}\right) \vee s_{1}=s_{2} \vee s_{1}=s_{1}$ by A2.

Definition 6: The binary priority operation $\geq$ on a substantive belief set $S$ is a belief term meet, if it satisfies the three conditions $C 1, C 2$, and $C 3$ supra and the pair $(S, \geq)$ then is a belief meet-semilattice.

\subsection{The belief term complete lattice}

For our intentions we will establish the following condition:

Condition 2: The set $S$ will be a finite set.

Consequence 2: Set $S$ has a supremum belief $s_{1}$ and an infimum belief $s_{n}$.

Consequence 3: All subset of $S$ also will be finite and has a supremum and an infimum belief.

Let $s_{i}$ and $s_{j}$ be two terms of $(\mathrm{S}, \geq)$ and $\mathrm{S}^{\prime}$ a subset of $\mathrm{S}$.

Definition 7: If for all elements $s_{i}$ and $s_{j}$, if $s_{i}$ is more than or equal to $s_{j}$ and $s_{j}$ is an element of $S^{\prime}$, then $s_{1}$ is also in $\left.S^{\prime}: \forall s_{i} \forall s_{j} \mid s_{i} \geq s_{j} \wedge s_{j} \in S^{\prime} \Rightarrow s_{i} \in S^{\prime}\right\rfloor$ then $S^{\prime}$ is the lower belief set or belief downward closed.

Let $s_{i}, s_{j}, s_{l} \in S$ be three terms of a belief poset $(S, \geq)$ and so that $S^{\prime}=\left\{s_{i}, s_{j}, s_{l}\right\} \subset S$.

Definition 8: The subset $S^{\prime}$ of a belief poset $(S, \geq)$ is called a directed belief subset if $S^{\prime}$ 'is not the empty set, and for any $s_{j}$ and $s_{l}$ in $S^{\prime}$ there exists a $s_{i}$ in $S^{\prime}$ with $s_{i} \geq s_{j}$ and $s_{j} \geq s_{l}$.

Consequence 4: All belief subset $S^{\prime}$ is directed.

Definition 9: The belief subset $S^{\prime}$ is a proper belief ideal, if the following conditions hold:

1) $S^{\prime}$ is a lower belief set: $\forall s_{i} \in S^{\prime}, s_{i} \geq s_{j} \Rightarrow s_{j} \in S^{\prime}$. 
2) S' is a directed belief set.

Definition 10: A belief ideal is a complete belief ideal if it is equal to the whole belief substantive set $S$.

Definition 11: The smallest belief ideal containing the supremum belief $s_{1}$ is a principal belief ideal and $s_{1}$ is said to be a principal term of the belief ideal in this situation. The principal belief ideal for a principal term $s_{1}$ is just given by the set $\left\{s_{i} \in S \mid s_{1} \geq s_{i}\right\}$.

Definition 12: A non-empty belief subset $S^{\prime \prime}$ of a belief poset $(S, \geq)$ is a proper belief filter if the following conditions hold:

1) $S$ "' is a belief filter base: For every $s_{i}, s_{j}$ in $S$ ', there is some element $s_{l}$ in $S^{\prime \prime}$, such that $s_{i} \geq s_{l}$ and $s_{j} \geq s_{l}$.

2) $S^{\prime \prime}$ is a belief upper set: For every $s_{i}$ in $S$ ', and $s_{j}$ in $S, s_{j} \geq s_{i}$ implies that $s_{j}$ is in $S^{\prime \prime}$.

Definition 13: A belief filter is a complete belief filter if it is equal to the whole belief substantive set $S$.

Definition 14: The smallest belief filter that contains an infimum belief $s_{n}$ is a principal belief filter and $s_{n}$ is a principal term in this situation. The principal belieffilter for $s_{n}$ is just given by the $\operatorname{set}\left\{x_{j} \in S \mid s_{j} \geq s_{n}\right\}$.

Definition 15: A belief poset $(S, \geq)$ is a complete poset because each of its beliefs subsets are directed (consequence 3 ) and has a belief supremum and a belief infimum.

An order in which all finite sets have both a supremum and an infimum is a lattice. In our case it will be a belief lattice.

Let us suppose a subset $S^{\prime}$ of the belief poset $(S, \geq)$ of substantive beliefs with a priority relation. A belief join of $S^{\prime}$ is a term of $S$ which is greater than or equal to every element of $S$ '. Formally, the belief meet of a subset $S$ ' of the belief poset is an element $s_{n}$ of $S$ such that

1) $\forall s_{i} \in S^{\prime}, s_{1} \geq s_{n}$.

2) $\forall s_{j} \in S$ if $\forall s_{i} \in S^{\prime}, s_{i} \geq s_{j}$, then $s_{i} \geq s_{n}$.

Definition 16: A belief subset $S$ ' of a belief lattice $(S, \geq)$ is a belief ideal iff it is a lower belief set that is closed under finite belief joins.

Definition 17: A belief subset $S^{\prime}$ of a belief lattice $(S, \geq)$ is a belief filter, iff it is an upper belief set that is closed under finite belief meets.

$S$ is said to satisfy the descending chain condition because every descending chain $s_{1} \geq s_{2} \geq s_{3} \geq \ldots \geq s_{n}$ of elements of $S$ there exists a positive integer $\mathrm{n}$ such that $s_{n}=s_{n+1}=s_{n+2}=\ldots$, there is no infinite descending chain.

Minimal condition: Every nonempty belief subset of $S$ has a minimal term. 
All chains have also a supremum belief. Therefore the belief lattice $(S, \geq)$ will be $\omega$ complete.

Consequence 5: The belief lattice $(S, \geq)$ is a complete lattice.

Consequence.6: The belief lattice $(S, \geq)$ fulfills the conditions of strong completeness.

\subsection{Belief uniformity}

Let $S=\left\{s_{1}, s_{2}, \ldots, s_{n}\right\}$ be the set of substantive beliefs and $S X S$ be its Cartesian product. Let $\Theta$ be a nonempty family of subsets of the Cartesian product $\Theta \subseteq S X S$ called the uniform structure or belief uniformity of $S$ and let $\mathrm{U}$ be a set so that $U \in \Theta$.

Definition 18: The elements of $\Theta$ are called b-entourages satisfying the following axioms:

1) Axiom 10: If $U$ is in $\Theta$, then $U$ contains the diagonal $\Delta=\left\{\left(s_{i}, s_{i}\right): s_{i} \in S\right\}$. Each term is $U$-close to itselffor each b-entourage $U$.

2) Axiom 11: If $U$ is in $\Theta$ and $V$ is a subset of $S X S$ which contains $U$, then $V$ is in $\Theta$.

3) Axiom 12: If $U$ and $V$ are in $\Theta$, then $U \cap V$ is in $\Theta$. Being both $U$-close and $V$ close is also a closeness relation in the uniformity.

4) Axiom 13: If $U$ is in $\Theta$, then there exists $V$ in $\Theta$ such that, whenever $\left(s_{1}, s_{2}\right),\left(s_{2}, s_{3}\right)$ are in $V$, then $\left(s_{1}, s_{3}\right)$ is in $U$. For each b-entourage $U$ there is a b-entourage $V$ which is half as large.

5) Axiom 14: If $U$ is in $\Theta$, then $U^{-1}=\left\{\left(s_{2}, s_{1}\right):\left(s_{1}, s_{2}\right)\right.$ in $\left.U\right\}$ is also in $\Theta$. It states the essentially symmetric property "closeness" with respect to a uniform structure.

It is easy to verify that the space $(S, \Theta)$ fulfills the previous axioms.

Definition 19: We define $s_{1}, s_{2}$ as U-close if $\left(s_{1}, s_{2}\right) \in U$.

The b-entourage $U$ is symmetric because $\left(s_{1}, s_{2}\right) \in U$ and $\left(s_{2}, s_{1}\right) \in U$. Every uniform belief space has a fundamental system of b-entourages consisting of symmetric bentourages.

Let

$$
\begin{aligned}
& \{\varnothing\},\left\{s_{1}\right\},\left\{s_{2}\right\}, \ldots,\left\{s_{n}\right\},\left\{\varnothing, s_{1}\right\}, \ldots,\left\{s_{1}, s_{2}\right\}, \ldots,\left\{s_{n-1}, s_{n}\right\}, \ldots,\left\{s_{1}, s_{2}, \ldots, s_{n}\right\}= \\
& S_{o}, S_{1}, S_{2}, \ldots, S_{n}, S_{01}, \ldots, S_{12}, \ldots, S_{(n-1) n}, \ldots, S
\end{aligned}
$$

be a collection of sets whose elements are substantive beliefs.

Definition 20: As $S \subset \bigcup_{\alpha \in A} S_{\alpha}$ we say that $C=\left\{S_{\alpha}: \alpha \in A\right\}$ is a belief cover of $S$.

Let $\mathrm{C}$ and $\mathrm{D}$ be two belief covers of $\mathrm{S}$. If every set in $\mathrm{D}$ is contained in some set in $\mathrm{D}$ we say that cover $\mathrm{D}$ is a refinement of belief cover $\mathrm{C}$. 
Definition 21: $D=V_{j \in J}$ is a refinement of $C=U_{i \in I}$ if $\forall j \exists i$ so that $V_{j} \subseteq U_{i}$.

\subsection{Metric Belief Space}

Let $S=\left\{s_{1}, s_{2}, \ldots, s_{n}\right\}$ be the set of substantive beliefs. A metric on a set $S$ is a function called the belief distance and so that $\delta: S x S \rightarrow \mathfrak{R}$ where $\mathfrak{R}$ is the set of real numbers.

Note 4: Belief distance $\delta$ is subjective and it depends on the believer.

Therefore metric $\delta$ will be a belief metric (b-metric).

For all $s_{1}, s_{2}, s_{3}$ in $S$, this function is required to satisfy the following conditions:

1) Non-negativity: $\delta\left(s_{1}, s_{2}\right) \geq 0$

2) Identity of indiscernibles: $\delta\left(s_{1}, s_{2}\right)=0$ iff $s_{1}=s_{2}$

3) Symmetry: $\delta\left(s_{1}, s_{2}\right)=\delta\left(s_{2}, s_{1}\right)$

4) Triangle inequality: $\delta\left(s_{1}, s_{3}\right) \leq \delta\left(s_{1}, s_{2}\right)+\delta\left(s_{2}, s_{3}\right)$

Therefore, we may define $\mathrm{S}$ like a metric belief space iff the believer subject defines a belief distance which will be always subjective.

Definition 22: The ordered pair $\Sigma=(S, \delta)$ is a metric belief space.

1) $\forall s_{i}, s_{2} \in S, \delta\left(s_{1}, s_{2}\right)=0$ iff $s_{1}=s_{2}$

2) $\forall s_{i}, s_{2}, s_{3} \in S, \delta\left(s_{1}, s_{3}\right) \leq \delta\left(s_{1}, s_{2}\right)+\delta\left(s_{2}, s_{3}\right)$

In a metric belief space

$$
\left\{\begin{array}{l}
\delta\left(s_{i}, s_{j}\right)=0 \text { if } s_{i}=s_{j} \\
\delta\left(s_{i}, s_{j}\right)=1 \text { otherwise }
\end{array}\right.
$$

Therefore, the metric belief space $\Sigma=(\mathrm{S}, \delta)$ has a discrete b-metric. This, in particular, shows that for any substantive belief set $\mathrm{S}$, there is always a metric belief space associated to it. Using this b-metric, any term is an open ball, and therefore every substantive belief subset $S_{i} \subseteq S$ is open and the metric belief space (S, $\delta$ ) has a discrete belief topology.

Let $s_{1}, s_{2}$ be two terms so that $s_{1}, s_{2} \in S$ and let $\mathrm{r}$ be a radius so that $r \in \Re, r>0$.

Definition 23: We define an open ball of radius $r>0$ centered at a term $s_{2}$ in $S$, to $B_{r}\left(s_{2}\right)^{\nabla}=\left\{s_{1} \in M \mid \delta\left(s_{1}, s_{2}\right)<r\right\}$

Definition 24: We define a closed ball of radius $r>0$ centered at a term $s_{2}$ in $S$, to $B_{r}\left(s_{2}\right)^{\nabla}=\left\{s_{1} \in M \mid \delta\left(s_{1}, s_{2}\right) \leq r\right\}$

Note 5: In any set of substantive beliefs $S$ any subject can make or construct as many open as closed balls. 
Note 6: By the peculiar characteristics of $S$, the balls will always be referred to term $s_{1}$ considered as the main term or supremum belief.

Let $S^{\prime}$ be a subset of a metric belief space $\Sigma=(S, \delta)$ such that $S^{\prime} \subseteq S$ and E be a size.

Definition 25: We say that $\mathrm{S}^{\prime}$ is bounded if there exists an $s_{1}$ in $S$ and $r>0$ such that for all $s_{i}$ in $S_{\Sigma}$, we have $\delta\left(s_{1}, s_{i}\right)<r$.

$\Sigma$ is a bounded metric belief space because $\Sigma$ is bounded as a subset of itself.

Definition 26: A subset $S^{\prime}$ of a metric belief space $\Sigma$ is a totally bounded set iff given size $E$, there exists a natural number $n$ and a family $S_{1}, S_{2}, \ldots, S_{n}$ of subsets of $S$, such that $S_{\Sigma}$ is contained in the union of the family, and such that each set $S_{i}$ in the family is of size $E$.

$$
\forall E, \exists n, \exists S_{1}, S_{2}, \ldots, S_{n} \subseteq S\left(S^{\prime} \subseteq \bigcup_{i=1}^{n} S_{i} \wedge \forall i=1, \ldots, n \text { size }\left(S_{i}\right) \leq E\right)
$$

Note 7: The metric belief space $\Sigma$ is a totally bounded belief space iff it is a totally bounded belief set when considered as a subset of itself $S \subseteq S$.

Note 8: $S$ is totally bounded if, given any positive radius $r>0$, it is covered by finitely many balls of radius $r$.

The absolute value $\left|s_{i}-s_{j}\right|$ can be replaced by the belief distance $\delta\left(s_{i}, s_{j}\right)$ between $\mathrm{s}_{\mathrm{i}}$ and $s_{j}$. It will allow us to establish Cauchy sequences in the metric belief space $\Sigma$.

Given a metric belief space $\Sigma=(\mathrm{S}, \delta)$, a belief sequence is Cauchy, if there exists a positive real $\varepsilon>0$ and there is a positive integer $N$ such that for all natural numbers $i, j>$ $N$, the belief distance $\delta\left(s_{i}, s_{j}\right)$ is less than $\varepsilon$. The terms of the belief sequence are getting closer and closer together in a way that suggests that the belief sequence ought to have a limit in $S$.

In a metric belief space $\Sigma=(S, \delta)$, the sets $U_{a}=\left\{\left(s_{1}, s_{2}\right) \in S X S \mid \delta\left(s_{1}, s_{2}\right)<a\right\}$ where a $>0$ form a fundamental system of b-entourages for the standard uniform structure of $S$. Then $s_{1}$ and $s_{2}$ are $U_{a}$-close precisely when the distance between $s_{1}$ and $s_{2}$ is at most $a$.

Definition 27: In a metric belief space $\Sigma=(S, \delta)$, a set $V$ is an uniform belief neighborhood of a term $p$ if there exists an open ball with centre $s$ and radius $r$, such that $B_{r}(p)=\{s \in S \mid d(s, p)<r\} \subset V$.

We may see in figure 2. 


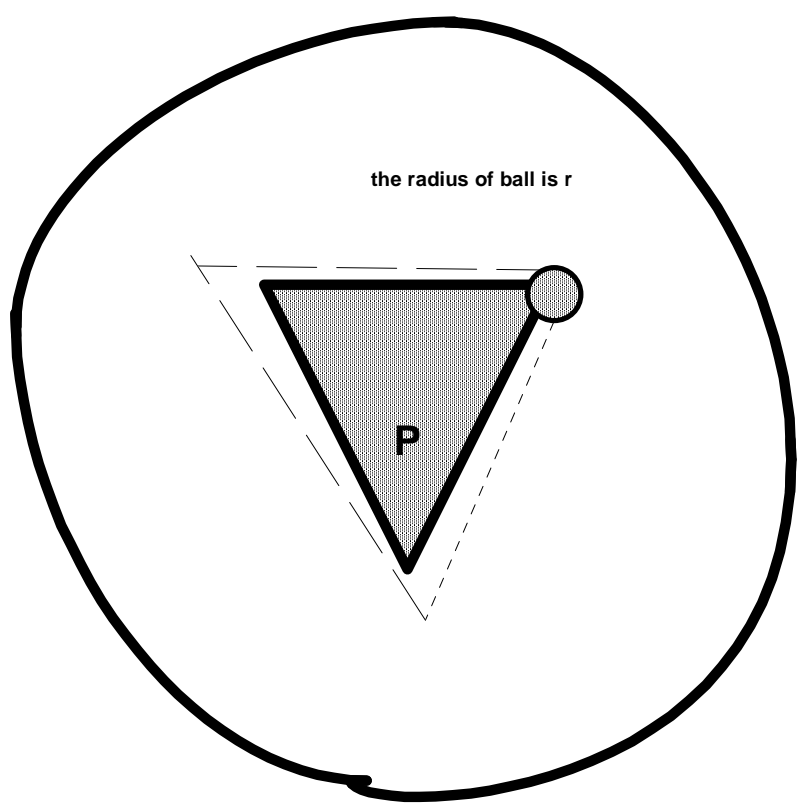

Figure 2: Metric belief space.

Definition 28: A pseudometric belief space $(S, \delta)$ is a set $S$ of substantive beliefs together with a non-negative real-valued function $\delta ; S X S \rightarrow \mathfrak{R}$ such that, for every

$s_{1}, s_{2}, s_{3} \in S$,

1) $\delta\left(s_{1}, s_{1}\right)=0$

2) $\delta\left(s_{1}, s_{2}\right)=\delta\left(s_{2}, s_{1}\right)=0$

3) $\delta\left(s_{1}, s_{3}\right) \leq \delta\left(s_{1}, s_{2}\right)+\delta\left(s_{2}, s_{3}\right)$

There is a metric identification, that converts the pseudometric belief space into a fullfledged metric belief space by defining $s_{1} s_{2}$ if $\delta\left(s_{1}, s_{2}\right)=0$. Let $S^{*}=S / \sim$ and let $\delta^{*}\left(\left[s_{1}\right],\left[s_{2}\right]\right)=\delta\left(s_{1}, s_{2}\right)$. Then $\delta^{*}$ is a belief metric on $S^{*}$ and $\left(S^{*}, \delta^{*}\right)$ is a welldefined metric belief space. Let $f: S X S \rightarrow \mathfrak{R}$ be a belief pseudometric on a set of substantive beliefs S. For a family $\left(f_{i}\right)$ of belief pseudometrics on $S$, the uniform structure defined by the family is the least upper belief bound of the uniform belief structures defined by the individual belief pseudometric $f_{\mathrm{i}}$. The family of belief pseudometrics is finite and it can be seen that the same belief uniform structure is defined by a single belief pseudometric, namely the upper belief envelope $\left(\sup f_{i}\right)$ of the family.

\subsection{The Topological Belief Space}

The open balls of a metric belief space $S$ form a basis for the topological belief space, whose open sets are all possible unions of open balls. This space is called the belief topology induced by the metric $d$.

Let $\mathrm{S}$ be a set of substantive beliefs and $\Theta$ be a collection of sets so that $\Theta=\left\{\varnothing,\left\{s_{1}\right\}, \ldots,\left\{s_{n}\right\},\left\{s_{i}, s_{2}\right\}, \ldots .\left\{s_{i}, s_{n}\right\}, \ldots, S\right\}$. The pair $(S, \Theta)$ will form a topological space because it fulfills the following conditions:

1) $\varnothing$ and $S$ are in $\Theta$.

2) The union of any collection of sets in $\Theta$ is also in $\Theta$. 
3) The intersection of any finite collection of sets in $\Theta$ is also in $\Theta$.

Definition 29: The topological space $(S, \Theta)$ we call the topological belief space.

Definition 30: The collection $\Theta$ is called a belief topology on $\mathrm{S}$ and the elements of $S$ are called substantive beliefs o terms.

Let $P(S)$ be the power set of $\mathrm{S}$ and $\sigma_{1}, \sigma_{2}$ be two sets so that $\sigma_{1}, \sigma_{2} \in P(S)$. We define the function $b c l: P(X) \rightarrow P(X)$ called the closure belief operator satisfying the following Kuratowski closure axioms:

1) Extensivity: $\sigma_{1} \subseteq \operatorname{bcl}\left(\sigma_{1}\right)$

2) Idempotence: $\operatorname{bcl}\left(\operatorname{bcl}\left(\sigma_{1}\right)\right)=\operatorname{bcl}\left(\sigma_{1}\right)$

3) Preservation of binary unions: $\operatorname{bcl}\left(\sigma_{1}\right) \cup b c l\left(\sigma_{2}\right)=\operatorname{bcl}\left(\sigma_{1} \cup \sigma_{2}\right)$

4) Preservation of nullary unions: $b c l(\varnothing)=\varnothing$

5) Preservation of finitary unions: $\operatorname{bcl}\left(\sigma_{1} \cup \sigma_{2} \cup \ldots \cup \sigma_{n}\right)=b c l\left(\sigma_{1}\right) \cup b c l\left(\sigma_{2}\right) \cup \ldots \cup b c l\left(\sigma_{n}\right)$

Then a topological belief space can be defined as $(S, b c l)$. Given a topological belief space $(S, \Theta)$ and a subset $S^{\prime} \subset S$, the belief subspace topology on $S^{\prime}$ is defined by $\Theta_{S^{\prime}}=\left\{S^{\prime} \cap X \mid X \in \Theta\right\}$.

Definition 31: If $S$ ' is equipped with the belief subspace topology then it is a topological belief space, and is called a belief subspace of $(S, \Theta)$.

Let $\Sigma^{\prime}$ and $\Sigma$ be two belief topologies on a belief set $S$ such that $\Theta^{\prime} \subseteq \Theta$, that is, every element of $\Theta^{\prime}$ is also an element of $\Theta$. Then the belief topology $\Theta^{\prime}$ is a coarser belief topology than $\Theta$, and $\Theta$ is said to be a finer belief topology than $\Theta^{\prime}$. If $\Theta^{\prime} \neq \Theta, \Theta^{\prime}$ is strictly coarser than $\Theta$ and $\Theta$ is strictly finer than $\Theta$ '.

If the set $S$ has a collection of subsets $\Theta$ that is a topological belief space then any member of $\Theta$ is an open set. We call B to topological belief space $B=(S, \Theta)=(S, b c l)$.

Let B be a topological belief space and $s \in S$ be a term. Let us suppose that $s \in U \subseteq V$. Then the set $\mathrm{V}$ will be a belief neighborhood of term $\mathrm{s}$ and $\mathrm{s}$ is in the interior of $\mathrm{V}$. The collection of neighborhoods of s will form a neighborhood filter $V(s)$ of term s. Let $\mathrm{V}$ be the neighborhood of $\mathrm{s}$ and let $\mathrm{B}$ a set such that $B \subset V$. There is a neighborhood filter $B(s) \subset V(s)$ such that $\forall V \in V(s), \exists B \in B(s) . B(s)$ is the local belief base the term $s$.

Let $S^{\prime}$ be a subset of topological belief space B such that $S^{\prime} \subset S$. The closure of $S^{\prime}$ consists in all terms (terms) which are close to $S^{\prime}$.

Definition 32: A term $s \in B$ is an adherent term for $S^{\prime}$ if every open set containing $s$ contains at least one term of $S$ ' other than $s$.

A term $s$ is an adherent term for $S^{\prime}$ iff $s$ is in the closure of $S^{\prime} \backslash\{s\}$. A term that is not an adherent term of $S^{\prime}$ is said to be an isolated term of $S^{\prime}$.

Definition 33: Term $s$ is a term of closure of $S^{\prime}$ if every neighborhood of $s$ contains a term of $S$ '. 
Consequence 7: Substantive beliefs have an abstract or ideal topological structure ${ }^{4}$.

\section{MATERIALIZATION OF BELIEF SYSTEMS}

Materialization is the conversion by means of certain mathematical correspondences, of an abstract set whose elements are beliefs or ideas, in an impure set whose elements are material or energetic. In their discussion on the materialization of ideology, DeMarrais et al. (1996) focused on understanding how the transformation of ideas, values, stories, myths and the like can be materialized into a physical reality that can take the form of ceremonial events, symbolic objects, monuments, and writing. The interest in the social engagement between people and material culture in ancient societies has resulted in numerous publications that seek to revise the original theoretical tenets proposed by DeMarrais and her colleagues (DeMarrais et al., 2004), as well as a renewed concern for the relationship between objects, social practices and human and non-human agency. Cognitive scientists have in fact noticed the importance of unique and unexpected events in framing the cognitive schemata of individuals through so-called flashbulb memories that concern our recall for the circumstances in which we learned of some significant event that, usually, was unexpected (McCauley \& Lawson 2002). Thus, importance should be given to a materialization of religious beliefs that stimulates the senses of the involved agents (Keane, 2008; Meyer, 2008) through the use of cues that are part of a system of settings and activities, and thereby give meaning to the built environment in which ritual practices are enacted and shared between religious specialists and ritual participants (Rapoport, $1990^{\mathrm{b}}$ ). According to Rapoport (1988), to understand the meaning of a built environment we have to interpret the relationship between the cues (i.e., fixed-features, semi-fixed and informal elements) that create a system of settings and activities (DeMarrais, 2004). It is the setting, with its sensorial aura, that is pivotal to structuring the ideological construct of the society, because it has both communicative and mnemonic functions, eliciting appropriate behavior by the participants (Rapoport, 1990 ). This theoretical framework is based on a nonverbal communication system in which all involved elements can be envisioned as nodes (Knappett, 2005); through the use of connecting ties (Jones, 2007), the nodes form a network that establishes the meanings of the material culture concerned with religious practices. The elements involved in the materialization of the network are diverse and combine a patchwork of sensorial experiences, i.e. visual, tactual, sound, smell, taste, that are interconnected by complex forms of ritual practices shared by the participants (Jones, 2007). Through the use of connecting ties (Jones, 2007), the nodes form a network that establishes the meanings of the material culture concerned with religious practices. For the involved cues to be both functional and meaningful, the context, the participants prior knowledge and the social practice involving human and non-human elements appear as central for a coherent and powerful construction of the meaning of materiality. In a similar way, Bell (1992) affirms that "ritual acts must be understood within a semantic framework whereby the significance of an action is dependent upon its place and relationship within a context of all other ways of acting: what it echoes, what it inverts, what it alludes to, what it denies".

\footnotetext{
${ }^{4}$ For a belief system to have a certain topological structure does not mean a different belief system has the same topology. Moreover, within the same belief system, just adding or subtracting a substantive belief does not mean that the topology will be different. But the laws of mathematics are always the same, not the consequences (materialization).
} 
In materialization process, we will distinguish two different although intimately united processes: symbolic materialization and textual materialization. We will divide to the Primigenial Base on two parts, $\mathrm{PB}_{1}$ containing the archetypes and $\mathrm{PB}_{2}$ containing myths (Figure 3).

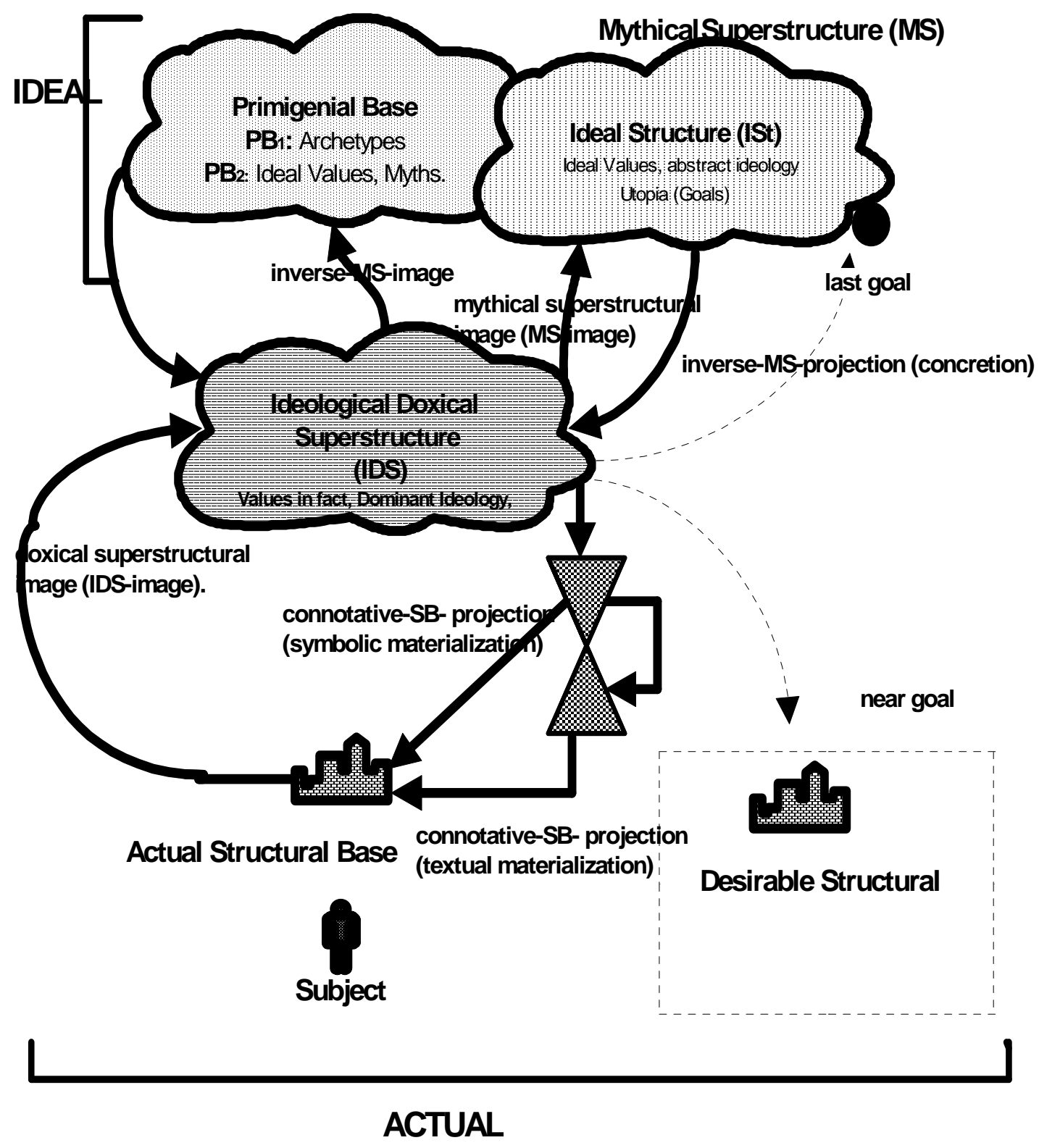

Figure 3: Mechanism for materialization.

\section{CONCLUSIONS}

We have sought to demonstrate that beliefs and their textual materialization have each topological structures. Nevertheless, from where do these mathematical structures come? In the case of structures of visual materialization the answer seems clear. However, it is not so in the world of beliefs and ideas. Unless we accept the philosophy of Plato, freeing the world of ideas from the human being, beliefs, both substantive and derived have a material origin, inserted in the human brain. This can be considered from two aspects: psychological and linguistic: 
1) Associated with the topological points is a family of open sets that cover space, like the response fields that constitute neighbourhoods of the actual neurons in the brain. The key point is that there are certain invariants associated with a topology that remain unchanged under the transformations. In the case of the visual field, the transformations are the distortions imposed by viewing conditions. The objects in the visual field are recognized as what they are in their own right no matter how their appearance may be distorted by viewing conditions: near or far, right-left, up-or-down in the field of view, rotated, moving, or viewed obliquely or binocularly. In addition, a tune is still recognizable even if it is shifted in key or changed in loudness, or heard biaurally. These invariance constitute the psychological constancies. Lacking constancy invariance, you would always be moving through a surrealistic world of perpetually deforming, rubbery objects. For the visual system, it is axiomatic that an object is determined by its bounding contours, and it is the invariance of these under different viewing conditions that determines constancy and form memory (Lewin, 1936). This brings us to the blessed domain of Lie transformation groups, denoted symbolically by the mapping $G X T \rightarrow G$, where

$\mathrm{G}$ is a mathematical group and $\mathrm{T}$ is a manifold (Text). $\mathrm{G}$ is also continuous and is a manifold just like space-time. Now think of a visual contour as a path-curve generated by the transformation group action, and choose some point on it. Call this the identity element of the group. Draw a tiny tangent line to the curve at that point. This is the infinitesimal transformation of the continuous or Lie group. The infinitesimal transformation is embodied in a Lie derivative, which "drags the flow along the path-curve", the so-called "orbit"--in this case the visual contour of $\mathrm{T}$. If $£$ denotes the Lie derivative and $\mathrm{f}$, the visual contour, then invariance of the contour under the transformation group is shown by its being annulled by the action of the Lie derivative: $£ \mathrm{f}=0$, or by its being handed on as a "contact element" for further processing: $£ f=g(f)$. These operations characterize psychological constancy.

2) In addition, neurological processes are organized and sequenced through language; hence, language reflects the way each person perceives the world. Being a psycho-biological process, one could say that mental maps are a sort of biological path along which words travel. The mental representations of individuals depend on their experiences, culture, ideology and physiology, among other things. Language refers to the way individuals make use of verbal expression to communicate experience, and this is done with the structure implicit in their own language.

Each of the substantive beliefs are propositions and these are formed by concepts. A concept is an analytical definition, an abstraction formed in the mind of a subject belonging to a particular semiotic system $S$. The different schools of philosophy have different contradictory views on the epistemological meaning of the concept. Here, the concept is the main component of rational thought in the attempt to apprehend reality. The concept is the union of denotative and connotative significances (Usó-Doménech and, 2012; Nescolarde-Selva and Usó-Doménech, 2013 ${ }^{\mathrm{a}, \mathrm{b}}$ ). And the connotative significance of a concept is different according to the World vision of any particular belief system. And not only that, but within a World vision, each individual may have a different connotation of the concept itself. Within the three monotheistic religions 
(Judaism, Christianity and Islam) the same concept of God has different connotations. For Judaism, God is the Absolute, is not represented and His Name is not known . YHVH, Adonai, HaShem, etc are just substitutes. And if He cannot be conceived in imagination, He can not be represented. Therefore, there is no religious art, as in the case of Christianity. And a narrow interpretation of the biblical words "man is made in the image and likeness of God" implies pictorial or sculptural human representation are wicked. Not until the nineteenth century do we find Jewish painters and sculptors. The case of Islam is not much different, although there are exceptions, such as the Iranian and Turkish miniatures, but in no case are there representations of divine characters as in the case of the Christian world where paintings, sculptures, etc., fill cathedrals, churches, monasteries, abbeys, museums.

All human experiences, as well as their expression through language, are subject to processes that may constitute evidence of failures in the world of vision, failures in the form of omissions, distortions, and generalizations. According to Cobb (1997), every individual has a particular way of relating and ordering perceived sequences of events that is captured through his conversations. This is because human beings communicate through a narrative language that has a time, a space, and a logic for building relationships, all of which is reflected as coherence. In conversing, human beings express the manner in which they relate things, but also the manner in which they relate to one another. This is done through words that express meanings.

In all materialization, human beings construct a sort of text $\mathrm{T}$ that may be understood as an analyzable object in which different structures may be identified, ranging from concrete organizations to abstract entities (Serrano, 2001). Meaning is built up through language; hence, the semantic value of the resulting text. Diverse orders exist:

1) Positional order: In an effort to give meaning and significance to the texts, human beings apply a variety of organization strategies, assigning to structures defined as semantic units, a relational order. This order (De Erice, 2002), may be a positional order, where language alignment is mediated by space-time variables (syntagmatic order).

2) Functional order, of codified association, since semantic units can only take on value related to others that may substitute it and constitute contextual relationships (paradigmatic order). There exists an ordering of text production and interpretation conditions in communication phenomena that goes beyond pragmatic factors to include situations of codified communication, inherited from culture, ideology and history.

3) Referential order: There is also a referential order that determines the influence of the linguistic over the non-linguistic strata in practice. In this manner, the interpretive path of a text $\mathrm{T}$ entails a series of operations that allow us to assign one or more meanings or senses to a linguistic series.

4) The hermeneutic order is the one guiding the production and interpretation of texts, that is to say, the one generating the content which is what has been defined as the text's plan, made from the set of meanings.

The interaction among different semantic units gives cohesion to a linguistic series, which is defined by its internal semantic relationships. However, the dynamic 
interaction also defines a coherence mediated by the relationships it establishes with its environment.

The specificity of a text $\mathrm{T}$ results from the intersection of a great number of structures which, when taken separately, are quite general. Nevertheless, experience shows that this is the point of view of the text, from a hermeneutic perspective, the one compelling the addition of contextual elements: without this, interpretation is incomplete and connotative comprehension unsatisfactory. In this manner the semantic process, the discourse, which is the set of codified linguistic uses together with a certain social practice - understood as the sphere of shared mental representations - defines a sort of associative network between units of meaning, which in their interactional dynamics define the context for reinterpreting the text $\mathrm{T}$.

A conceptual map is a graphic mental representation of a network of semantic units whose interactions define a context of meanings (denotations and connotations).

The object of conceptual maps is to represent meaningful relationships between concepts in the form of propositions. A proposition consists of two or more concepts joined by linking words to form a semantic unit, that is, a unit with meaning. For Novak \& Gowin (1984), a conceptual map "can provide a kind of visual road map showing some of the pathways we may take to connect meanings of concepts in propositions". Several authors have stated that conceptual maps are networks of semantic relationships, where semantic refers to the meaning or interpretation of the meaning which individuals attribute to a given symbol, word, language or other formal representation. It is during this negotiation (which may take place with others, but also with oneself), if done conscientiously, that individuals may come to recognize the generalizations, omissions, and distortions contained in their texts, and restructure their narratives. All modification of cognitive structure reports in the terminology of neurolinguistic theory, a new mental WV. This is the importance of conceptual maps.

These theoretical arguments seem useful for analyzing and understanding results obtained by Miller and Cañas (2008), which indicate a relationship between the topological and semantic aspects of conceptual maps. The topological taxonomy classifies conceptual maps according to five criteria:

1) Concept recognition.

2) Presence of linking phrases.

3) Degree of ramification.

4) Depth.

5) Presence of cross-links.

These criteria consider progressively more complex topological entities, beginning with concepts, passing through propositions, beliefs, etc. and ending with a complete conceptual map (Figure 4). 


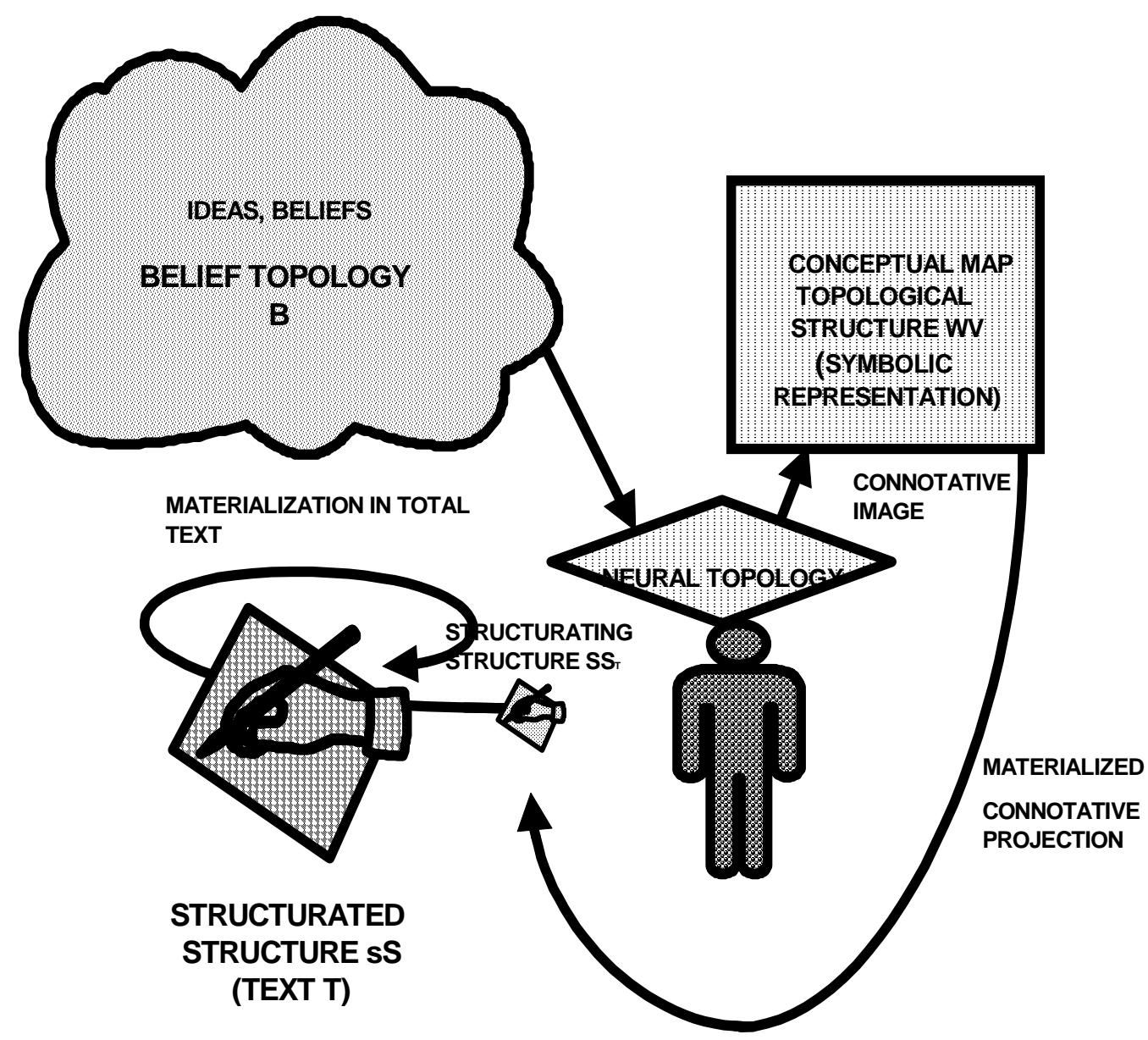

Figure 4: The conceptual map.

The mechanism is the following one:

1) Once beliefs (nodes) have been placed in a map, they are related to one another to form larger graphic structures by means of any form of symbolic representation - this is the linking phrase.

2) Ramification occurs when several relationships emanate from the same node or make use of the same linking element.

3) Hierarchical depth refers to the number of levels of beliefs nested under the root (main) concept of the map.

Though this nesting may indeed be evidence of conceptual subsumption, the two are not to be confused; this topological criterion considers only the number of the level, not what concepts are placed in each of them. The last criterion deals with cross-links. From the perspective of spatial organization, cross-links, when accompanied by all the other elements mentioned above, lead to topological entities of greater overall complexity. Therefore, this would appear to be a semantic criterion. However, the ability to recognize individual concepts and beliefs is so basic to being able to build up rich, interconnected, flexible conceptual map topologies that this criterion is included among the structural criteria. In other words, the focus is not on what is actually said, but on 
whether the mapper is able to recognize beliefs in their original context and depict the way in which they are related to one another.

As Novak \& Gowin (1984) have noted, "Conceptual mapping has been developed specifically to tap into a learner's cognitive structure and to externalize...what the learner already knows". Although conceptual maps certainly do not provide a "complete representation of the relevant concepts and propositions a learner knows... [they do constitute] a workable approximation". This is the main argument that shows the relationship between topology and semantics, between graphical configuration and meaning and it implies a dynamic relationship between the topological and semantic aspects of conceptual maps, where the former may be conceived as the dependent variable, and the latter as the independent one. Being a dynamic interaction, in giving expression to a text in a conceptual map the dependent variable helps to reorganize the independent variable.

This would explain why it is stated that there are no good or bad conceptual maps; it is the reason why it is said that the conceptual map represents the state of a subject's knowledge on the topic at a given moment. The topological-semantic relationship would seem to be led by semantics. In other words, changes on the semantic front give rise to changes on the topological front. Changes in topology however have little influence upon semantics, but do offer important information that can provide feedback to the subject to help produce changes in his cognitive structure, that is, to learn in a meaningful way.

From the viewpoint of the neurolinguistic model, each person is said to have a mental world vision WV in which his life unfolds. This representation is called the individual's mental world vision (MWV), which in turn becomes expressed through texts T.

The conceptual map is a text representing meanings, is a reflection of the person's connotation, of the way the person communicates with himself and with others.

When that communication is to be represented graphically through a conceptual map, its physical layout or configuration reflects the way he or she arranges sequences of relationships makes differentiations and identifies or discovers integrations, all of which serve to construct meanings. However, this spatial aspect of a conceptual map depends on the content with which the subject interprets the world and its relationships.

Neurolinguistics, from its practical approach states that by generating changes in an individual's language, changes in his mental model can be achieved and a new model will generate new behaviours.

In the learning process if a change in the cognitive structure occurs, with new words, new symbols, new beliefs and new representations, with the intention of obtaining new meanings within that frameset, the consequence will be a shift in individuals' emotional state, responses and behaviours.

Human beings utilize certain cognitive strategies to integrate coherence and cohesion into meanings. These information organization strategies are generalizations, distortion and elimination of data. For this reason, neurolinguistic theory (Nescolarde-Selva and Usó-Domènech. $2013^{c}$ ) considers it indispensable that individuals acquire the ability to recognize their generalizations, to recover the parts omitted from their model of the world, and to correct its distortions, in order to guide in a precise way the process of shifting their mental models. In that new context, mediated by new communications, underlying mental models are modified, and consequently changes are produced in semantic processes. This requires a new organization, which shows up in a conceptual map as changes in topological structure. As a consequence, we establish the following hypothesis: Changes in the semantic structure of a conceptual map generate changes in the topological structure. 


\section{Acknowledgment}

The authors would like to thank Dr. Hugh Gash for his important collaboration in this work, especially English corrections and scientific recommendations.

\section{REFERENCES}

Anderson, I. 1987. Combinatorics of Finite Sets. Oxford University Press. Oxford. England.

Andreas, H. 2011. A Structuralist Theory of Belief Revision. Journal of Logic, Language and Information. Volume 20, Issue 2, pp 205-232.

Bell, C., 1992. Ritual Theory, Ritual Practice. Oxford: Oxford University Press.

Birkhoff, G. 1967. Lattice Theory, 3rd ed. Amer. Math. Soc. Providence, RI.

Bourbaki, N. 1972. Commutative Algebra. (English translation ed.). Addison-Wesley.

Borhek, J.T. and Curtis, R.F. 1983. A Sociology of Belief. Robert E. Krieger Publishing Company. Malabar. Florida.

Bredon, G. E. 1997. Topology and Geometry. Graduate Texts in Mathematics. Springer.

Brewka, G. 1991. Belief Revision in a Framework for Default Reasoning. In: Proceedings of the Workshop on The Logic of Theory Change. Springer, London, 602622.

Bryant, V. 1985. Metric Spaces: Iteration and Application. Cambridge University Press. Burris, S. N., and Sankappanavar, H. P. 1981. A Course in Universal Algebra. SpringerVerlag.

Cobb, S. 1997. Una perspectiva narrativa de la mediación. Editorial Piados. México. (In Spanish).

De Erice, M.V.G. 2002. Pequeño Glosario de Semántica. Traducción. Universidad Nacional de Cuyo. (In Spanish).

DeMarrais, E., 2004. The materialization of culture. In: Rethinking Materiality: the Engagement of Mind with the Material World. Eds. E. DeMarrais, C. Gosden \& C. Renfrew. (McDonald Institute Monographs.) Cambridge: McDonald Institute for Archaeological Research, 11-22.

DeMarrais, E., L.J. Castillo \& T. Earle, 1996. Ideology, materialization, and power strategies. Current Anthropology 37, 15-31.

DeMarrais, E., C. Gosden \& C. Renfrew, 2004. Rethinking Materiality: the Engagement of Mind with the Material World. McDonald Institute Monographs. Cambridge: 
Durkheim, E. 2001. The elementary forms of the religious life. Oxford University Press.

Durkheim, E.and Mauss, M. 1963. Primitive Classification. University of Chicago Press.

Eliade, M. 1978. A history of Religious Ideas. Volume I: From the Stone Age to the Eleusinian Mysteries. University of Chicago Press. Chicago.

Ferreras, J.I. 1980. Fundamentos de Sociología de la Literatura. Ediciones Cátedra, SA. Madrid. (In Spanish).

Geertz, C. 1973. The Interpretation of Cultures. Basic Books.

Hansson, S. O. 1999. A Textbook of Belief Dynamics. Theory Change and Database Updating. Kluwer, Dordrecht.

Harrod, J.B. 1992. Two Millions Years Ago: The Origins of Art and Symbol. Continuum. 2, 1. 4-29.

Jones, A., 2007. Memory and Material Culture. (Topics in Contemporary Archaeology) Cambridge: Cambridge University Press.

Keane, W. 2008. The evidence of the sense and the materiality of religion. Journal of the Royal Anthropological Institute 14 (S1), s110-27.

Kelley, J. L. 1955. General Topology. D. Van Nostrand Company, Inc. Princeton, NJ.

Klüver, J., 2011. A mathematical theory of communication: Meaning, information, and topology. Complexity. Vol. 16. Issue 3. pp: 10-26.

Knappett, C., 2005. Thinking Through Material Culture: an Interdisciplinary Perspective. Philadelphia (PA): University of Pennsylvania Press.

Lakoff, G. 1987. Women, Fire and Dangerous Things: What Categories Reveal about the Mind. The University of Chicago Press. Chicago.

Levi-Strauss, Claude. 1963. The Structural Study of Myth. Structural Anthropology. Vol. 1. Trans. Clair Jacobson and Brooke Grundfest Shoepf. New York.Basic,. 206-231.

Lewin, K. 1936. Principles of Topological Psychology. New York and London. McGraw-Hill.

McCauley, R.N. \& E.T. Lawson, 2002. Bringing Ritual to Mind: Psychological Foundations of Cultural Forms. Cambridge: Cambridge University Press.

Meyer, B., 2008. Media and senses in the making of religious experience: an introduction. Material Religion 4(2), 124-35.

Miller, N. L.and Cañas, A. J. 2008. A semantic scoring rubric for conceptual maps: design and reliability. In: A. J. Cañas, P. Reiska, M. Åhlberg \& J. D. Novak (Eds.). 
Conceptual mapping: Connecting Educators. Proceedings of the Third International Conference on Conceptual mapping, Tallinn, Estonia \& Helsinki, Finland.

Munkres, J. 1999. Topology. Prentice Hall.

Nescolarde-Selva, J. and Usó-Domènech, J.L. 2013ª . Semiotic Vision of Ideologies. Foundations of Science. DOI 10.1007/s10699-013-9329-9.

Nescolarde-Selva, J. and Usó-Doménech, J. 2013 ${ }^{\mathrm{b}}$ Reality, System and Impure Systems. Foundations of Science. DOI: 10.1007/s10699-013-9337-8.

Nescolarde-Selva, J. and Usó-Doménech, J. 2013 ${ }^{\mathrm{c}}$ Model, Metamodel and Topology. Foundations of Science. DOI 10.1007/s10699-013-9334-y.

Nöth, W. 1994. Der Text als Raum. In: Dieter W. Halwachs et al. (eds.). Sprache, Onomatopöie, Rhetorik, Namen, Idiomatik, Grammatik. Festschrift für Karl Sornig. Graz Universität. 163-174.. (In German).

Novak, J. D., \& Gowin, D. B. 1984. Learning how to learn. Cambridge University Press. New York .

Peirce, C. S. 1958. Collected Papers of Charles Sanders Peirce, vols. 1-8, C. Hartshorne, P. Weiss y A. W. Burks (eds.). Cambridge, MA: Harvard University Press.

Rapoport, A., 1988. Levels of meaning in the built environment. In Cross-Cultural Perspectives in Nonverbal Communication, Ed. F. Poyatos. Lewiston (NY): Hogrefe, 317-33.

Rapoport, A., $1990^{\mathrm{a}}$. The Meaning of the Built Environment: a Nonverbal Communication Approach. 2nd edition. Tucson (AZ): The University of Arizona Press.

Rapoport, A., $1990^{\mathrm{b}}$. Systems of activities and systems of settings, In Domestic Architecture and the Use of Space. an Interdisciplinary Cross-Cultural Study, Ed. S. Kent. Cambridge: Cambridge University Press, 9-20.

Rott, H. 2001. Change, Choice and Inference: A Study of Belief Revision and Nonmonotonic Reasoning. Oxford University Press, Oxford.

Rudin, M. E. 1969. A new proof that metric spaces are paracompact. Proceedings of the American Mathematical Society, Vol. 20, No. 2. p. 603.

Serrano, E. 1999. Semiótica Verbal 5: Discurso, texto y contexto. (Translation: Jacques Fontanille). In: Sémiotique et littérature. Essai de méthode. PUF . Paris, pp. 16-18. 2001. (in Spanish).

Schechter E. 1997. Handbook of Analysis and Its Foundations. Academic Press.

Scholem, G. 1941. Major Trends in Jewish Mysticism. Schocken Books. New York. 
Swanson, G.E. 1964. The Birth of the Gods. The Origin of the Primitive Beliefs. Ann Arbor Paperback.

Usó-Domènech, J.L., Vives-Maciá, F., Nescolarde Selva, J. and Patten, B.C. 2009 ${ }^{\text {a }}$ A Walford 's metadynamic point of view of ecosustainability ideology (I). INTERSYMP 2009.

Usó-Domènech, J.L., Vives-Maciá, F., Nescolarde Selva, J. and Patten, B.C. 2009 ${ }^{\text {. A }}$ Walford 's metadynamic point of view of ecosustainability ideology (II). INTERSYMP 2009.

Usó-Doménech, J.L. and Nescolarde-Selva, J. 2012. Mathematic and semiotic theory of ideological systems. Editorial LAP. Saarbrücken. Germany.

Walsby, H. 1947. The Domain of Ideologies: a study of the origin, structure and development of ideologies. Wm.McLellan in Collaboration with the Social Science Association. Glasgow

Wenz, K. 1997. Kultur als Programm. Textuelle Raumzeichen und textueller Zeichenraum als Ereignis. Kodikas Supplement Series 22. Tubingen. (In German).

Willard, S. 1970. General Topology. Addison-Wesley Publishing Company, Reading Massachusetts.

Willard, S. 2004. General Topology. Dover Publications. 